$\exists \int$ Journal of Logic \& Analysis 3:4 (2011) 1-15

ISSN 1759-9008

\title{
Uniform liftings of continuous mappings
}

\author{
ALEXANDER P PYSHCHEV
}

\begin{abstract}
We investigate the question of when a continuous mapping between subspaces of nonstandard hulls has a uniform lifting.

2000 Mathematics Subject Classification 03H05, 54J05 (primary); 54C20, 54D20, 54D65 (secondary)
\end{abstract}

Keywords: nonstandard analysis, nonstandard hull, uniform lifting

\section{Introduction}

Let $\mathcal{H}$ and $\mathcal{H}^{\prime}$ be (some) nonstandard hulls of *metric spaces $(M, d)$ and $\left(M^{\prime}, d^{\prime}\right)$ respectively and $\mathcal{M}$ a subspace of $\mathcal{H}$. Following Fajardo and Keisler [6, Definition 4.14], we say that a mapping $f: \mathcal{M} \rightarrow \mathcal{H}^{\prime}$ is uniformly liftable if there exists an internal mapping $\phi: M \rightarrow M^{\prime}$ such that for every equivalence class $\mu \in \mathcal{M}$ the image $\phi[\mu]$ is contained in $f(\mu)$; the mapping $\phi$ in this case is said to be a uniform lifting of $f$. It is easily checked that every uniformly liftable mapping is continuous. The converse is not true:

Example 1.1 Let $d$ be a $\{0,1\}$-valued ${ }^{*}$ metric on ${ }^{*} \mathbb{N}$ and let

$$
\mathcal{H}=\left\{\{x\}: x \in{ }^{*} \mathbb{N}\right\}
$$

be the nonstandard hull of $\left({ }^{*} \mathbb{N}, d\right)$. (Here and in the following $\mathbb{N}$ denotes the set of nonnegative integers.) It is clear that the mapping $f: \mathcal{H} \rightarrow \mathcal{H}$ defined by $f(\{x\})=$ $\left\{\chi_{\mathbb{N}}(x)\right\}$, where $\chi_{\mathbb{N}}:{ }^{*} \mathbb{N} \rightarrow\{0,1\}$ is the characteristic function of $\mathbb{N}$, is continuous but not uniformly liftable.

By [5, Proposition 4.12] and [6, Corollary 4.8, Theorem 4.18] every continuous mapping $f: \mathcal{M} \rightarrow \mathcal{H}^{\prime}$ defined on a compact set $\mathcal{M}$ is uniformly liftable. More generally, we have the following result: (We assume that our nonstandard universe $\left(V(\Xi), V\left({ }^{*} \Xi\right), *\right)$ is $\kappa$-saturated, where $\kappa$ is an uncountable cardinal.) 
Proposition 1.2 Let $f: \mathcal{M} \rightarrow \mathcal{H}^{\prime}$ be a continuous mapping from a subspace $\mathcal{M}$ of the nonstandard hull $\mathcal{H}$ of a ${ }^{*}$ metric space $(M, d)$ around $c \in M$ into the nonstandard hull $\mathcal{H}^{\prime}$ of a *metric space $\left(M^{\prime}, d^{\prime}\right)$ around $c^{\prime} \in M^{\prime}$. If $\mathcal{M}$ has a dense subspace of cardinality $<\kappa$ and $|\mathcal{M}|<|V(\Xi)|$, then $f$ is uniformly liftable.

Proof Assume first that $f$ is an isometric embedding of $\mathcal{M}$ into $\mathcal{H}^{\prime}$. Let $D$ be a dense subspace of $\mathcal{M}$ of cardinality $<\kappa$. For every $\mu \in D$ choose $x_{\mu} \in \mu$ and $y_{\mu} \in f(\mu)$. For every $(\mu, n) \in D \times \mathbb{N}$ let $\Psi_{\mu, n}$ be an internal set consisting of all internal mappings $\psi: H \rightarrow M^{\prime}$ with the following properties:

(i) $H$ is a hyperfinite subset of $M$ containing $x_{\mu}$;

(ii) $\psi\left(x_{\mu}\right)=y_{\mu}$;

(iii) $\left|d^{\prime}(\psi(x), \psi(y))-d(x, y)\right|<1 /(n+1)$ for all $(x, y) \in H^{2}$.

If $F \subseteq D$ is finite, then the mapping $\xi_{F}:\left\{x_{\mu}: \mu \in F\right\} \rightarrow M^{\prime}, \xi_{F}\left(x_{\mu}\right)=y_{\mu}$, is in $\Psi_{\mu, n}$ for every $(\mu, n) \in F \times \mathbb{N}$. By $\kappa$-saturation there exists a mapping $\psi_{0}: H_{0} \rightarrow M^{\prime}$ which belongs to the intersection of all $\Psi_{\mu, n}$. Since $D$ is dense in $\mathcal{M}$, by $\omega_{1}$-saturation $H_{0}$ intersects with every $\mu \in \mathcal{M}$. Since $H_{0}$ is hyperfinite, by transfer there exists an internal mapping $r: M \rightarrow H_{0}$ such that

$$
d(x, r(x))=\min \left\{d(x, y): y \in H_{0}\right\}
$$

for every $x \in M$; it follows that $x \in \mu \in \mathcal{M}$ implies $r(x) \in \mu$. It is easily checked that $\phi=\psi_{0} \circ r$ is a uniform lifting of some mapping $g: \mathcal{M} \rightarrow \mathcal{H}^{\prime}$. Since $g$ is uniformly liftable, it is continuous. Mappings $f$ and $g$ coincide on $D$, and since $D$ is dense in $\mathcal{M}$, we have $f=g$. Thus $\phi$ is a uniform lifting of $f$.

Now we turn to the general case. Since $|\mathcal{M}|<|V(\Xi)|$, there exist isometries $h$ : $\mathcal{M} \rightarrow X$ and $h^{\prime}: f[\mathcal{M}] \rightarrow X^{\prime}$, where metric spaces $X$ and $X^{\prime}$ are in $V(\Xi)$. Let $e$ and $e^{\prime}$ denote the canonical embeddings of $X$ and $X^{\prime}$ into their nonstandard hulls. Let $\psi: M \rightarrow{ }^{*} X$ be a uniform lifting of an isometry $e \circ h: \mathcal{M} \rightarrow e[X]$. Since $f$ is continuous, $f[\mathcal{M}]$ has a dense subspace of cardinality $<\kappa$. Therefore, there exists a uniform lifting $\tilde{\psi}:{ }^{*} X^{\prime} \rightarrow M^{\prime}$ of an isometry $\left(e^{\prime} \circ h^{\prime}\right)^{-1}: e^{\prime}\left[X^{\prime}\right] \rightarrow f[\mathcal{M}]$. It is clear that

$$
\phi=\tilde{\psi} \circ *\left(h^{\prime} \circ f \circ h^{-1}\right) \circ \psi
$$

is a uniform lifting of $f$.

The condition $|\mathcal{M}|<|V(\Xi)|$ in Proposition 1.2 is redundant, see Theorem 1.8 below.

See Henson and Moore [11] and Baratella and $\mathrm{Ng}$ [2] for some related results in the context of nonstandard hulls of *normed spaces. 
Our goal is to extend Proposition 1.2 to a more general class of spaces that includes other types of nonstandard hulls, in particular, nonstandard hulls of topological vector spaces [10]. Following Gordon [7], we consider topological spaces obtained as subspaces of quotients of internal sets by $\Pi_{1}^{0}(\kappa)$ equivalence relations. (A set is said to be $\Pi_{1}^{0}(\kappa)$ (resp. $\Pi_{1}^{0}$ ) if it is the intersection of $<\kappa$ (resp. $\leq \omega$ ) internal sets.) If $R$ is a $\Pi_{1}^{0}(\kappa)$ equivalence relation on internal set $M$, then for every $\mathcal{M} \subseteq M / R$ and every $Z \subseteq M$ we put

$$
\begin{gathered}
O_{\mathcal{M}}(Z)=\{\mu \in \mathcal{M}: \mu \subseteq Z\}, \\
\operatorname{st}_{\mathcal{M}}(Z)=\{\mu \in \mathcal{M}: \mu \cap Z \neq \emptyset\} .
\end{gathered}
$$

An easy argument shows that

$$
\left\{O_{M / R}(T): T \subseteq M \text { is internal }\right\}
$$

is the base of some Hausdorff topology on $M / R$. (Note that all equivalence classes $\mu \in M / R$ are $\Pi_{1}^{0}(\kappa)$ and apply $\kappa$-saturation.) We call this topology the canonical topology on $M / R$. It follows from Proposition 2.4 below that this topology is generated by some family of pseudometrics and hence is Tychonoff.

Example 1.3 Let $\left(\mathcal{H}, d_{c}^{\circ}\right)$ be the nonstandard hull of *metric space $(M, d)$ around $c \in M$. Then

$$
R=d^{-1}\left(\mu_{\mathbb{R}}(0)\right)=\bigcap_{n \in \mathbb{N}}\left\{(x, y) \in M^{2}: d(x, y)<1 /(n+1)\right\}
$$

is a $\Pi_{1}^{0}$ equivalence relation on $M$ and $\mathcal{H} \subseteq M / R$. We claim that the topology generated by $d_{c}^{\circ}$ coincides with that induced by the canonical topology on $M / R$. Fix $\mu \in \mathcal{H}$ and $x \in \mu$; put

$$
\begin{gathered}
b(x, n)=\{y \in M: d(x, y)<1 /(n+1)\}, \\
B(\mu, n)=\left\{\nu \in \mathcal{H}: d_{c}^{\circ}(\mu, \nu)<1 /(n+1)\right\} .
\end{gathered}
$$

If $T \subseteq M$ is internal and $\mu \subseteq T$, then by $\omega_{1}$-saturation there exists $n \in \mathbb{N}$ so that $\mu \subseteq b(x, n) \subseteq T$, and it follows that $\mu \in B(\mu, n) \subseteq O_{M / R}(T) \cap \mathcal{H}$. On the other hand, $\mu \in O_{M / R}(b(x, n+1)) \subseteq B(\mu, n)$ for every $n \in \mathbb{N}$, and the proof of our claim is complete.

Example 1.4 Let $(K, \tau)$ be a compact Hausdorff space, $K \in V(\Xi)$. Assume that there exists a base $\mathcal{B}$ of topology $\tau$ of cardinality $<\kappa$. It is well-known that ${ }^{*} K$ is the disjoint union of monads $\mu_{K}(x), x \in K$; let $R=\bigcup_{x \in K} \mu_{K}(x)^{2}$ be the corresponding 
equivalence relation. Let $P$ denote the family of all pairs $(U, V) \in \mathcal{B}^{2}$ such that cl $U \subseteq V$, where cl $U$ is the closure of $U$ in $K$. We have

$$
R=\bigcap_{(U, V) \in P}{ }^{*} V^{2} \cup^{*}(K \backslash \operatorname{cl} U)^{2},
$$

therefore, $R$ is $\Pi_{1}^{0}(\kappa)$. It is easily checked that the mapping $e$ defined by $e(x)=\mu_{K}(x)$ is a homeomorphism of $K$ onto ${ }^{*} K / R$ endowed with the canonical topology.

Example 1.5 Let $(X, \tau)$ be a Tychonoff space, $X \in V(\Xi)$. Assume that there exists a base $\mathcal{B}$ of topology $\tau$ of cardinality $<\kappa$. By [4, Theorem 3.5.2] there exists a compact Hausdorff space $\left(K, \tau^{\prime}\right)$ containing $(X, \tau)$ as a subspace and such that there exists a base of topology $\tau^{\prime}$ of cardinality $<\kappa$. By [4, Theorem 3.5.3] we may also assume that $K \in V(\Xi)$. By the previous example $R_{K}=\bigcup_{x \in K} \mu_{K}(x)^{2}$ is $\Pi_{1}^{0}(\kappa)$, therefore, the equivalence relation $R=R_{K} \cap{ }^{*} X^{2}$ on ${ }^{*} X$ is also $\Pi_{1}^{0}(\kappa)$. For every $x \in X$ we have $\mu_{X}(x)={ }^{*} X \cap \mu_{K}(x)$, hence $\mu_{X}(x) \in{ }^{*} X / R$. As in the case of compact spaces, the mapping $e: X \rightarrow{ }^{*} X / R, e(x)=\mu_{X}(x)$, is a homeomorphism of $X$ onto $\left\{\mu_{X}(x): x \in X\right\}$.

Example 1.6 Let $E \in V(\Xi)$ be a vector space (over $\mathbb{F}=\mathbb{R}$ or $\mathbb{C}$ ) and $\tau$ a Hausdorff topology on $E$ such that $(E, \tau)$ is a topological vector space. Assume that the filter of neighborhoods of $0 \in E$ has a base $\mathcal{B}_{0}$ of cardinality $<\kappa$. Then

$$
R=\left\{(x, y) \in{ }^{*} E^{2}: x-y \in \mu_{E}(0)\right\}=\bigcap_{U \in \mathcal{B}_{0}}\left\{(x, y) \in{ }^{*} E^{2}: x-y \in{ }^{*} U\right\}
$$

is a $\Pi_{1}^{0}(\kappa)$ equivalence relation on ${ }^{*} E$. The nonstandard hull of $(E, \tau)$ is a topological vector space $(\hat{E}, \hat{\tau})$ defined by

$$
\hat{E}=\left\{\mu \in{ }^{*} E / R: \mu \subseteq \operatorname{fin}\left({ }^{*} E\right)\right\},
$$

where

$$
\text { fin }\left({ }^{*} E\right)=\left\{x \in{ }^{*} E: \epsilon x \in \mu_{E}(0) \text { for every } \epsilon \in \mu_{\mathbb{F}}(0)\right\} ;
$$

the filter of neighborhoods of $\mu_{E}(0)$ in $\hat{E}$ is generated by the sets $\operatorname{st}_{\hat{E}}\left({ }^{*} U\right), U \in \mathcal{B}_{0}$, see Henson [10]. It is easily checked that the nonstandard hull topology $\hat{\tau}$ coincides with that induced by the canonical topology on ${ }^{*} E / R$. (Apply Lemma 2.5 below.)

For further examples see Luxemburg [12], Henson [9], Gordon [7, 8], Mlček and Zlatoš [13], and Ziman and Zlatoš [14].

Let $R$ (resp. $R^{\prime}$ ) be a $\Pi_{1}^{0}(\kappa)$ equivalence relation on an internal set $M\left(\right.$ resp. $M^{\prime}$ ) and $f$ a mapping from $\mathcal{M} \subseteq M / R$ into $M^{\prime} / R^{\prime}$. We say that a mapping $\phi: Z \rightarrow M^{\prime}$ is 
a weak uniform lifting of $f$ if $\phi$ is an internal mapping with $Z=\operatorname{dom} \phi \subseteq M$ such that $\mu \cap Z \neq \emptyset$ and $\phi[\mu \cap Z] \subseteq f(\mu)$ for every $\mu \in \mathcal{M}$. A mapping $\phi$ is said to be a uniform lifting of $f$ if $\phi$ is a weak uniform lifting of $f$ with $\operatorname{dom} \phi=M$. We say that $f$ is (weakly) uniformly liftable if there exists a (weak) uniform lifting of $f$. Every weakly uniformly liftable mapping is continuous, see Proposition 4.2 below.

Gordon [7, Theorem 1.2] proved that if $R$ is a $\Pi_{1}^{0}(\kappa)$ equivalence relation on an internal set $M$ such that $M / R$ is compact, then every continuous mapping $f: M / R \rightarrow$ fin $\left({ }^{*} \mathbb{C}\right) / \approx$ is uniformly liftable.

We say that a topological space is $\kappa$-separable if it has a dense subspace of cardinality $<\kappa$.

The following are our main results:

Theorem 1.7 Let $R$ (resp. $R^{\prime}$ ) be a $\Pi_{1}^{0}(\kappa)$ equivalence relation on internal set $M$ (resp. $M^{\prime}$ ) and $\mathcal{M}$ a $\kappa$-separable subspace of $M / R$. Then every continuous mapping $f: \mathcal{M} \rightarrow M^{\prime} / R^{\prime}$ is weakly uniformly liftable. Moreover, a weak uniform lifting $\phi: H \rightarrow M^{\prime}$ of $f$ can be chosen so that $H=\operatorname{dom} \phi$ is a hyperfinite subset of $M$.

Theorem 1.8 Let $R$ (resp. $R^{\prime}$ ) be a $\Pi_{1}^{0}(\kappa)$ equivalence relation on internal set $M$ (resp. $M^{\prime}$ ) and $\mathcal{M}$ a subspace of $M / R$. Assume that at least one of the following conditions is satisfied:

(1) $\mathcal{M}$ is $\kappa$-separable and $R$ is $\Pi_{1}^{0}$;

(2) $\mathcal{M}$ is Lindelöf and $R^{\prime}$ is $\Pi_{1}^{0}$;

(3) $\mathcal{M}$ is compact and $\mathcal{M}=M / R$;

(4) $\mathcal{M}$ is separable and metrizable.

Then every continuous mapping $f: \mathcal{M} \rightarrow M^{\prime} / R^{\prime}$ is uniformly liftable.

Also we show that each of the following conditions (in the context of Theorem 1.8) is not sufficient for the existence of uniform liftings:

(i) $\mathcal{M}$ is separable and $f: \mathcal{M} \rightarrow{ }^{*}[0,1] / \approx$ is continuous;

(ii) $\mathcal{M}$ is separable and compact and $f: \mathcal{M} \rightarrow \mathcal{M}^{\prime}$ is a homeomorphism;

see Example 4.6 below. 


\section{Generating families of *pseudometrics}

Let $X$ be an arbitrary set and let $U$ and $V$ be the subsets of $X^{2}$. Recall the following definitions:

$$
\begin{gathered}
U^{-1}=\left\{(x, y) \in X^{2}:(y, x) \in U\right\}, \\
U[x]=\{y \in X:(x, y) \in U\},
\end{gathered}
$$

$V \circ U=\left\{(x, z) \in X^{2}\right.$ : there exists $y \in X$ so that $(x, y) \in U$ and $\left.(y, z) \in V\right\}$,

$$
U^{(1)}=U, \quad U^{(n+1)}=U^{(n)} \circ U, \quad n \geq 1 .
$$

By $\Delta_{X}$ we denote the diagonal of $X$.

Lemma 2.1 Let $X$ be a set and $V_{0}=X^{2}, V_{1}, V_{2}, \ldots$ a sequence of sets such that $\Delta_{X} \subseteq V_{n}=V_{n}^{-1} \subseteq X^{2}$ and $V_{n+1}^{(3)} \subseteq V_{n}$ for all $n \geq 1$. Then there exists a pseudometric $d$ on $X$ such that $d^{-1}\left(\left[0,1 / 2^{n}\right)\right) \subseteq V_{n} \subseteq d^{-1}\left(\left[0,1 / 2^{n}\right]\right)$ for all $n \geq 1$.

Proof This is an easy corollary of [4, Theorem 8.1.10].

Let $R$ be an equivalence relation on internal set $M$ and $\left\{d_{\alpha}: \alpha \in A\right\}$ a family of *pseudometrics on $M$. We say that $R$ is generated by $\left\{d_{\alpha}: \alpha \in A\right\}$ and that $\left\{d_{\alpha}: \alpha \in A\right\}$ is a generating family for $R$ if

$$
R=\left\{(x, y) \in M^{2}: d_{\alpha}(x, y) \approx 0 \text { for every } \alpha \in A\right\} .
$$

Proposition 2.2 Every $\Pi_{1}^{0}(\kappa)$ equivalence relation $R$ on internal set $M$ has a generating family $\left\{d_{\alpha}: \alpha \in A\right\}$ of *pseudometrics on $M$ such that $|A|<\kappa$.

Proof Let $\mathcal{U}$ be an internal set consisting of all internal sets $U$ such that $\Delta_{M} \subseteq U=$ $U^{-1} \subseteq M^{2}$. Let $\left\{U_{\alpha}: \alpha \in A\right\} \subseteq \mathcal{U}$ be such that $|A|<\kappa$ and $R=\bigcap_{\alpha \in A} U_{\alpha}$. We may assume that $\left\{U_{\alpha}: \alpha \in A\right\}$ is closed under finite intersections. Clearly, $R \subseteq \bigcap_{\alpha \in A} U_{\alpha}^{(2)}$. On the other hand, if $(x, y) \in U_{\alpha}^{(2)}$ for all $\alpha \in A$, then by $\kappa$-saturation

$$
R[x] \cap R[y]=\bigcap_{(\alpha, \beta) \in A^{2}} U_{\alpha}[x] \cap U_{\beta}[y] \neq \emptyset,
$$

hence $(x, y) \in R$. It follows that $R=\bigcap_{\alpha \in A} U_{\alpha}^{(2)}$. By $\kappa$-saturation for every $\alpha \in A$ there exists $\beta \in A$ such that $U_{\beta}^{(2)} \subseteq U_{\alpha}$. Hence for every $\alpha \in A$ there exists $\gamma \in A$ such that $U_{\gamma}^{(3)} \subseteq U_{\gamma}^{(4)} \subseteq U_{\alpha}$. Let $f: A \rightarrow A$ be such that $U_{f(\alpha)}^{(3)} \subseteq U_{\alpha}$ for every $\alpha \in A$. 
Fix $\alpha \in A$. By $\omega_{1}$-saturation there exists an internal sequence $\left(V_{\nu}\right)_{0 \leq \nu \leq H}$ consisting of elements of $\mathcal{U}$ such that $H \in{ }^{*} \mathbb{N} \backslash \mathbb{N}, V_{0}=M^{2}, V_{1}=U_{\alpha}, V_{n+1}=U_{f^{(n)}(\alpha)}$ for every $n \in \mathbb{N} \backslash\{0\}$, and $V_{\nu+1}^{(3)} \subseteq V_{\nu}$ for every $\nu<H$. Put $V_{\nu}=\Delta_{M}$ for all $\nu>H$. By transfer of Lemma 2.1 there exists a ${ }^{*}$ pseudometric $d_{\alpha}$ on $M$ such that

$$
d_{\alpha}^{-1}\left(\left[0,1 / 2^{\nu}\right)_{*}\right) \subseteq V_{\nu} \subseteq d_{\alpha}^{-1}\left(\left[0,1 / 2^{\nu}\right] * \mathbb{R}\right), \quad \nu \geq 1 .
$$

We have $R \subseteq d_{\alpha}^{-1}\left(\mu_{\mathbb{R}}(0)\right) \subseteq U_{\alpha}$. It is clear that $R$ is generated by the family $\left\{d_{\alpha}: \alpha \in A\right\}$.

Proposition 2.3 Every $\Pi_{1}^{0}$ equivalence relation on internal set $M$ is generated by some *metric $d$ on $M$.

Proof Applying Proposition 2.2 with $\kappa=\omega_{1}$, we obtain a generating family $\left\{d_{n}\right.$ : $n \in \mathbb{N}\}$ for $R$. We may assume that all * pseudometrics $d_{n}$ take values in * $[0,1]$ (e.g., we may consider a ${ }^{*}$ pseudometric $\bar{d}_{n}(x, y)=\min \left\{d_{n}(x, y), 1\right\}$ instead of $\left.d_{n}\right)$. Let $\left(d_{\nu}\right)_{0 \leq \nu \leq H}$ be an internal extension of $\left(d_{n}\right)_{n \in \mathbb{N}}$ which also consists of *pseudometrics on $M$ with values in ${ }^{*}[0,1]$. We may assume that $d_{H}$ is a $\{0,1\}$-valued ${ }^{*}$ metric on M. Put

$$
d(x, y)=\sum_{\nu=0}^{H} d_{\nu}(x, y) / 2^{\nu+1} .
$$

Clearly, $d^{-1}\left(\mu_{\mathbb{R}}(0)\right) \subseteq R$. On the other hand, if $(x, y) \in R$, then by $\omega_{1}$-saturation there exists $K \in{ }^{*} \mathbb{N} \backslash \mathbb{N}, K<H$, so that $\sum_{\nu=0}^{K} d_{\nu}(x, y) / 2^{\nu+1} \approx 0$; since then

$$
\sum_{\nu=K+1}^{H} d_{\nu}(x, y) / 2^{\nu+1} \leq 1 / 2^{K+1} \approx 0,
$$

we obtain $d(x, y) \approx 0$.

Assume that an equivalence relation $R$ on internal set $M$ is generated by the family $\left\{d_{\alpha}: \alpha \in A\right\}$ of *pseudometrics. For every $\alpha \in A$ define $d_{\alpha}^{\circ}:(M / R)^{2} \rightarrow[0, \infty]$ by

$$
d_{\alpha}^{\circ}(\mu, \nu)={ }^{\circ}\left(d_{\alpha}(x, y)\right), \quad x \in \mu, y \in \nu ;
$$

it is easily checked that $d_{\alpha}^{\circ}$ is a $[0, \infty]$-valued pseudometric on $M / R$.

Proposition 2.4 Let $R$ be a $\Pi_{1}^{0}(\kappa)$ equivalence relation on internal set $M$ and $\left\{d_{\alpha}\right.$ : $\alpha \in A\}$ a generating family of ${ }^{*}$ pseudometrics for $R$ with $|A|<\kappa$. Then the topology on $M / R$ generated by pseudometrics $d_{\alpha}^{\circ}, \alpha \in A$, coincides with the canonical topology. 
Proof Fix $x \in \mu \in M / R$ and put

$$
\begin{gathered}
b(x, n, \alpha)=\left\{y \in M: d_{\alpha}(x, y)<1 /(n+1)\right\}, \\
B(\mu, n, \alpha)=\left\{\nu \in M / R: d_{\alpha}^{\circ}(\mu, \nu)<1 /(n+1)\right\} .
\end{gathered}
$$

If $\mu \subseteq T$, where $T$ is an internal subset of $M$, then since

$$
\mu=\bigcap_{(n, \alpha) \in \mathbb{N} \times A} b(x, n, \alpha) \subseteq T,
$$

by $\kappa$-saturation there exists a finite set $F \subseteq \mathbb{N} \times A$ with $\bigcap_{(n, \alpha) \in F} b(x, n, \alpha) \subseteq T$. It follows that

$$
\mu \in \bigcap_{(n, \alpha) \in F} B(\mu, n, \alpha) \subseteq O_{M / R}(T)
$$

On the other hand, for any finite $F \subseteq \mathbb{N} \times A$ we have

$$
\mu \in O_{M / R}\left(\bigcap_{(n, \alpha) \in F} b(x, n+1, \alpha)\right) \subseteq \bigcap_{(n, \alpha) \in F} B(\mu, n, \alpha) .
$$

The result follows.

Lemma 2.5 Let $R$ be a $\Pi_{1}^{0}(\kappa)$ equivalence relation on internal set $M$ and $U$ an open in $M / R$ set containing $\mu \in M / R$. Then there exists an internal set $T$ such that $\mu \subseteq T \subseteq M$ and $\mathrm{st}_{M / R}(T) \subseteq U$.

Proof Easy (see the proof of Proposition 2.4).

Lemma 2.6 Let $R$ (resp. $R^{\prime}$ ) be a $\Pi_{1}^{0}(\kappa)$ equivalence relation on internal set $M$ (resp. $\left.M^{\prime}\right)$. Then

$$
R \otimes R^{\prime}=\left\{\left(\left(x, x^{\prime}\right),\left(y, y^{\prime}\right)\right) \in\left(M \times M^{\prime}\right)^{2}:(x, y) \in R \text { and }\left(x^{\prime}, y^{\prime}\right) \in R^{\prime}\right\}
$$

is a $\Pi_{1}^{0}(\kappa)$ equivalence relation on $M \times M^{\prime}$ and the mapping $h:\left(\mu, \mu^{\prime}\right) \mapsto \mu \times \mu^{\prime}$ is a homeomorphism of $(M / R) \times\left(M^{\prime} / R^{\prime}\right)$ onto $\left(M \times M^{\prime}\right) /\left(R \otimes R^{\prime}\right)$.

Proof Let $\left\{d_{\alpha}: \alpha \in A\right\}$ and $\left\{d_{\beta}^{\prime}: \beta \in B\right\}$ be generating families of *pseudometrics for $R$ and $R^{\prime}$ respectively with $|A|<\kappa$ and $|B|<\kappa$. Put

$$
\begin{gathered}
\rho_{\alpha}\left(\left(x, x^{\prime}\right),\left(y, y^{\prime}\right)\right)=d_{\alpha}(x, y), \quad \alpha \in A, \\
\rho_{\beta}^{\prime}\left(\left(x, x^{\prime}\right),\left(y, y^{\prime}\right)\right)=d_{\beta}^{\prime}\left(x^{\prime}, y^{\prime}\right), \quad \beta \in B .
\end{gathered}
$$


Since $R \otimes R^{\prime}$ is generated by $\left\{\rho_{\alpha}: \alpha \in A\right\} \cup\left\{\rho_{\beta}^{\prime}: \beta \in B\right\}$, it is $\Pi_{1}^{0}(\kappa)$. Using Proposition 2.4 and noting that

$$
\begin{gathered}
\rho_{\alpha}^{\circ}\left(\mu \times \mu^{\prime}, \nu \times \nu^{\prime}\right)=d_{\alpha}^{\circ}(\mu, \nu), \quad \alpha \in A, \\
\rho_{\beta}^{\circ}\left(\mu \times \mu^{\prime}, \nu \times \nu^{\prime}\right)=d_{\beta}^{\prime \circ}\left(\mu^{\prime}, \nu^{\prime}\right), \quad \beta \in B,
\end{gathered}
$$

it is easy to check that $h$ is a homeomorphism.

The following important lemma will be used without mentioning:

Lemma 2.7 Let $\mathcal{M} \subseteq(M / R) \cap(M / \tilde{R})$, where $R$ and $\tilde{R}$ are $\Pi_{1}^{0}(\kappa)$ equivalence relations on internal set $M$. Then the canonical topologies on $M / R$ and $M / \tilde{R}$ induce the same topology on $\mathcal{M}$.

Proof Note that $\mathcal{M} \cap O_{M / R}(T)=O_{\mathcal{M}}(T)=\mathcal{M} \cap O_{M / \tilde{R}}(T)$ for every internal $T \subseteq$ $M$.

\section{$3 \kappa$-Separable subspaces and weak uniform liftings}

Lemma 3.1 Let $R$ be a $\Pi_{1}^{0}(\kappa)$ equivalence relation on internal set $M$ and $\mathcal{M}$ a subspace of $M / R$. If $\mathcal{M}$ is Lindelöf, then it is $\kappa$-separable. If $\mathcal{M}$ is compact, then $\bigcup \mathcal{M}=\bigcup_{\mu \in \mathcal{M}} \mu$ is a $\Pi_{1}^{0}(\kappa)$ set and there exists a hyperfinite set $H \subseteq M$ such that $\mathcal{M}=\mathrm{st}_{M / R}(H)$.

Proof Assume that $\mathcal{M}$ is Lindelöf (resp. compact). Let $\left\{U_{\alpha}: \alpha \in A\right\}$ be a family of internal subsets of $M^{2}$ such that $|A|<\kappa$ and $R=\bigcap_{\alpha \in A} U_{\alpha}$. We may assume that $U_{\alpha}^{-1}=U_{\alpha}$ for every $\alpha \in A$ and that $\left\{U_{\alpha}: \alpha \in A\right\}$ is closed under finite intersections. Choose $x_{\mu} \in \mu$ for every $\mu \in \mathcal{M}$. For every $\alpha \in A$ the sets $O_{\mathcal{M}}\left(U_{\alpha}\left[x_{\mu}\right]\right), \mu \in \mathcal{M}$, are open in $\mathcal{M}$ and cover $\mathcal{M}$, therefore, there exists a countable (resp. finite) set $\mathcal{C}_{\alpha} \subseteq \mathcal{M}$ with $\mathcal{M}=\bigcup_{\mu \in \mathcal{C}_{\alpha}} O_{\mathcal{M}}\left(U_{\alpha}\left[x_{\mu}\right]\right)$.

Put $D=\bigcup_{\alpha \in A} \mathcal{C}_{\alpha}$; note that $D$ has cardinality $<\kappa$. We claim that $D$ is dense in $\mathcal{M}$. Fix $\mu \in \mathcal{M}$ and an open in $\mathcal{M}$ neighborhood $U$ of $\mu$. By Lemma 2.5 there exists an internal set $T \subseteq M$ such that $\mu \subseteq T$ and st $_{\mathcal{M}}(T) \subseteq U$. By $\kappa$-saturation there exists $\alpha \in A$ such that $U_{\alpha}\left[x_{\mu}\right] \subseteq T$. There exists $\nu \in \mathcal{C}_{\alpha}$ with $\mu \in O_{\mathcal{M}}\left(U_{\alpha}\left[x_{\nu}\right]\right)$. It follows that $x_{\nu} \in U_{\alpha}\left[x_{\mu}\right]$ and hence $\nu \in U$. Thus $\mathcal{M}$ is $\kappa$-separable.

Now we assume that $\mathcal{M}$ is compact. For every $\alpha \in A$ put $W_{\alpha}=\bigcup_{\mu \in \mathcal{C}_{\alpha}} U_{\alpha}\left[x_{\mu}\right]$. Clearly, $\bigcup \mathcal{M} \subseteq W_{\alpha}$. Since $\mathcal{C}_{\alpha}$ is finite, $W_{\alpha}$ is internal. We claim that $\bigcup \mathcal{M}=$ 
$\bigcap_{\alpha \in A} W_{\alpha}$. Let $y \in \nu \in(M / R) \backslash \mathcal{M}$. Since $\mathcal{M}$ is closed in $M / R$, by Lemma 2.5 and $\kappa$-saturation there exists $\alpha \in A$ so that $U_{\alpha}[y] \cap \bigcup \mathcal{M}=\emptyset$, hence $y \notin W_{\alpha}$. Thus $\bigcup \mathcal{M}$ is $\Pi_{1}^{0}(\kappa)$.

Since $\left\{x_{\mu}: \mu \in D\right\} \subseteq \bigcup \mathcal{M},|D|<\kappa$, and $\bigcup \mathcal{M}$ is $\Pi_{1}^{0}(\kappa)$, by $\kappa$-saturation there exists a hyperfinite set $H$ with $\left\{x_{\mu}: \mu \in D\right\} \subseteq H \subseteq \bigcup \mathcal{M}$. Then $\operatorname{st}_{M / R}(H)$ is a closed subset of $M / R$ such that $D \subseteq$ st $_{M / R}(H) \subseteq \mathcal{M}$; since $D$ is dense in $\mathcal{M}$, $\mathrm{st}_{M / R}(H)=\mathcal{M}$.

If $T$ and $S$ are internal sets, then by $S_{\text {int }}^{T}$ we denote the internal set consisting of all internal mappings $\phi: T \rightarrow S$.

Lemma 3.2 Let $T$ be an internal set and $A$ a nonempty subset of $T$ of cardinality $<\kappa$. Then the space ${ }^{*}[0,1]_{\text {int }}^{T} / R$, where $R$ is generated by *pseudometrics $d_{\alpha}(x, y)=$ $|x(\alpha)-y(\alpha)|, \alpha \in A$, is compact.

Proof We claim that ${ }^{*}[0,1]_{\text {int }}^{T} / R$ is homeomorphic to $[0,1]^{A}$. Clearly, the mapping

$$
w:{ }^{*}[0,1]_{\text {int }}^{T} \rightarrow[0,1]^{A}, \quad(w(x))(\alpha)={ }^{\circ}(x(\alpha)),
$$

induces an injection $h:{ }^{*}[0,1]_{\text {int }}^{T} / R \rightarrow[0,1]^{A}$. By $\kappa$-saturation $h$ is onto. The usual topology on $[0,1]^{A}$ is generated by pseudometrics $\rho_{\alpha}(f, g)=|f(\alpha)-g(\alpha)|$, $\alpha \in A$, and since $d_{\alpha}^{\circ}(\mu, \nu)=\rho_{\alpha}(h(\mu), h(\nu))$, by Proposition 2.4 mapping $h$ is a homeomorphism.

Theorem 3.3 Let $\mathcal{M}$ be a $\kappa$-separable subspace of $M / R$, where $R$ is a $\Pi_{1}^{0}(\kappa)$ equivalence relation on internal set $M$. Then there exists a $\Pi_{1}^{0}(\kappa)$ equivalence relation $\tilde{R}$ on $M$ such that $M / \tilde{R}$ is compact and $\mathcal{M} \subseteq M / \tilde{R}$.

Proof Let $\left\{d_{\alpha}: \alpha \in A\right\}$ be a generating family of *pseudometrics for $R$ such that $|A|<\kappa$ (see Proposition 2.2). We may assume that all $d_{\alpha}$ take values in ${ }^{*}[0,1]$. Let $D$ be a dense subspace of $\mathcal{M}$ of cardinality $<\kappa$. Choose $x_{\mu} \in \mu$ for every $\mu \in D$ and put $f_{\alpha, \mu}(x)=d_{\alpha}\left(x, x_{\mu}\right)$. Let $\tilde{R}$ be a $\Pi_{1}^{0}(\kappa)$ equivalence relation on $M$ generated by *pseudometrics

$$
\sigma_{\alpha, \mu}(x, y)=\left|f_{\alpha, \mu}(x)-f_{\alpha, \mu}(y)\right|, \quad(\alpha, \mu) \in A \times D .
$$

Put $\mathcal{F}={ }^{*}[0,1]_{\text {int }}^{M}$. Let $R^{\prime}$ be a $\Pi_{1}^{0}(\kappa)$ equivalence relation on ${ }^{*}[0,1]_{\text {int }}^{\mathcal{F}}$ generated by *pseudometrics

$$
\rho_{\alpha, \mu}(\phi, \chi)=\left|\phi\left(f_{\alpha, \mu}\right)-\chi\left(f_{\alpha, \mu}\right)\right|, \quad(\alpha, \mu) \in A \times D .
$$


By Lemma $3.2 \mathcal{K}={ }^{*}[0,1]_{\text {int }}^{\mathcal{F}} / R^{\prime}$ is compact.

Let $\theta: M \rightarrow{ }^{*}[0,1]_{\text {int }}^{\mathcal{F}}$ be an internal mapping defined by $(\theta(x))(f)=f(x)$. Then $\mathcal{K}_{0}=\operatorname{st}_{\mathcal{K}}(\theta[M])$ is a closed subspace of $\mathcal{K}$ and therefore is compact. Note that $\sigma_{\alpha, \mu}(x, y)=\rho_{\alpha, \mu}(\theta(x), \theta(y))$ for every $(\alpha, \mu) \in A \times D$. Using Proposition 2.4, it is easy to check that $\theta$ induces a homeomorphism of $M / \tilde{R}$ onto $\mathcal{K}_{0}$. It follows that $M / \tilde{R}$ is compact.

In order to prove that $\mathcal{M} \subseteq M / \tilde{R}$, note that $(x, y) \in R$ implies $d_{\alpha}\left(x, x_{\mu}\right) \approx d_{\alpha}\left(y, x_{\mu}\right)$ and hence $\sigma_{\alpha, \mu}(x, y) \approx 0$ for every $(\alpha, \mu) \in A \times D$. On the other hand, if $x \in \mu \in \mathcal{M}$ and $y \in M \backslash \mu$, then $d_{\alpha}(x, y)>\epsilon$ for some $\alpha \in A$ and $\epsilon \in \mathbb{R}, \epsilon>0$; since $D$ is dense in $\mathcal{M}$, there exists $\mu \in D$ such that $d_{\alpha}\left(x, x_{\mu}\right)<\epsilon / 3$; then $d_{\alpha}\left(x_{\mu}, y\right)>\epsilon / 2$; therefore, as $\sigma_{\alpha, \mu}(x, y) \not \approx 0$, we have $(x, y) \notin \tilde{R}$.

Corollary 3.4 Let $\mathcal{M}$ be a separable subspace of some nonstandard hull $\mathcal{H}$ of a ${ }^{*}$ metric space $(M, d)$. Then there exists a ${ }^{*}$ metric $\tilde{d}: M^{2} \rightarrow{ }^{*}[0,1]$ on $M$ such that the nonstandard hull $\tilde{\mathcal{H}}$ of $(M, \tilde{d})$ is compact and $\mathcal{M} \subseteq \tilde{\mathcal{H}}$.

Proof Put $\kappa=\omega_{1}$ in Theorem 3.3 and apply Propositions 2.3 and 2.4.

Corollary 3.5 Let $R$ be a $\Pi_{1}^{0}(\kappa)$ equivalence relation on internal set $M$ and $\mathcal{M}$ a $\kappa$-separable subspace of $M / R$. Then for every closed in $\mathcal{M}$ set $\mathcal{A} \subseteq \mathcal{M}$ there exists a hyperfinite set $H \subseteq M$ such that $\operatorname{st}_{\mathcal{M}}(H)=\mathcal{A}$.

Proof This follows easily from Theorem 3.3 and Lemma 3.1.

Proof of Theorem 1.7 Since $\mathcal{M}$ is $\kappa$-separable and $f$ is continuous, $f[\mathcal{M}]$ is also $\kappa$-separable. By Theorem 3.3 there exist $\Pi_{1}^{0}(\kappa)$ equivalence relations $\tilde{R}$ and $\tilde{R}^{\prime}$ on $M$ and $M^{\prime}$ respectively such that $\mathcal{M} \subseteq M / \tilde{R}, f[\mathcal{M}] \subseteq M^{\prime} / \tilde{R}^{\prime}$, and such that the spaces $M / \tilde{R}$ and $M^{\prime} / \tilde{R}^{\prime}$ are compact. Let $F$ be the closure of $f$ in $(M / \tilde{R}) \times\left(M^{\prime} / \tilde{R}^{\prime}\right)$; since $f$ is continuous, we have $F \cap\left(\mathcal{M} \times\left(M^{\prime} / \tilde{R}^{\prime}\right)\right)=f$. Since $F$ is compact, by Lemmas 2.6 and 3.1 there exists a hyperfinite set $\tilde{H} \subseteq M \times M^{\prime}$ such that

$$
\tilde{H} \subseteq \bigcup_{\left(\mu, \mu^{\prime}\right) \in F} \mu \times \mu^{\prime}
$$

and $\tilde{H} \cap\left(\mu \times \mu^{\prime}\right) \neq \emptyset$ for every $\left(\mu, \mu^{\prime}\right) \in F$. Let $H=\operatorname{dom} \tilde{H}$. By transfer there exists an internal mapping $\phi: H \rightarrow M^{\prime}$ such that $(x, \phi(x)) \in \tilde{H}$ for every $x \in H$; it is clear that $\phi$ is a weak uniform lifting of $f$. 


\section{Uniform liftings}

Proposition 4.1 Let $R$ (resp. $R^{\prime}$ ) be a $\Pi_{1}^{0}(\kappa)$ equivalence relation on internal set $M$ (resp. $M^{\prime}$ ), $\mathcal{M}$ a subspace of $M / R$, and $f: \mathcal{M} \rightarrow M^{\prime} / R^{\prime}$ a mapping. Consider the following statements:

(1) $f$ is uniformly liftable;

(2) there exists an extension $\tilde{f}: M / R \rightarrow M^{\prime} / R^{\prime}$ of $f$ which is continuous at every $\mu \in \mathcal{M}$.

We have (1) $\Rightarrow(2)$ and if $M / R$ and $M^{\prime} / R^{\prime}$ are compact, then (1) and (2) are equivalent.

Proof Assume (1). Let $\phi: M \rightarrow M^{\prime}$ be a uniform lifting of $f$. Put

$$
\Phi=\left\{\left(\mu, \mu^{\prime}\right) \in(M / R) \times\left(M^{\prime} / R^{\prime}\right): \phi \cap\left(\mu \times \mu^{\prime}\right) \neq \emptyset\right\} ;
$$

note that $\operatorname{dom} \Phi=M / R$ and $\Phi \cap\left(\mathcal{M} \times\left(M^{\prime} / R^{\prime}\right)\right)=f$. Let $\tilde{f}: M / R \rightarrow M^{\prime} / R^{\prime}$ be an arbitrary mapping such that $\tilde{f} \subseteq \Phi$. Clearly, $\tilde{f}$ is an extension of $f$. We claim that $\tilde{f}$ is continuous at every $\mu \in \mathcal{M}$. Let $U^{\prime}$ be an open neighborhood of $f(\mu)$. By Lemma 2.5 there exists an internal set $T^{\prime} \subseteq M^{\prime}$ such that $f(\mu) \subseteq T^{\prime}$ and $\mathrm{st}_{M^{\prime} / R^{\prime}}\left(T^{\prime}\right) \subseteq U^{\prime}$. Put $T=\phi^{-1}\left[T^{\prime}\right]$. Then $U=O_{M / R}(T)$ is an open neighborhood of $\mu$. Fix $\nu \in U$. There exists $x \in \nu$ such that $\phi(x) \in \tilde{f}(\nu)$. We have $x \in T$, therefore, $\phi(x) \in T^{\prime}$ and $\tilde{f}(\nu) \in U^{\prime}$.

Assume (2) and that $M / R$ and $M^{\prime} / R^{\prime}$ are compact. Let $F$ be the closure of $\tilde{f}$ in $(M / R) \times\left(M^{\prime} / R^{\prime}\right)$. Since $\tilde{f}$ is continuous at every $\mu \in \mathcal{M}$, we have $F \cap\left(\mathcal{M} \times\left(M^{\prime} / R^{\prime}\right)\right)=$ $f$. By Lemmas 2.6 and 3.1 the set

$$
\Pi=\bigcup_{\left(\mu, \mu^{\prime}\right) \in F} \mu \times \mu^{\prime}
$$

is $\Pi_{1}^{0}(\kappa)$. Since $\operatorname{dom} \Pi=M$, by $\kappa$-saturation there exists an internal mapping $\phi: M \rightarrow M^{\prime}$ such that $\phi \subseteq \Pi$. It is easily checked that $\phi$ is a uniform lifting of $f$.

Proposition 4.2 Let $R$ (resp. $\left.R^{\prime}\right)$ be a $\Pi_{1}^{0}(\kappa)$ equivalence relation on internal set $M$ (resp. $M^{\prime}$ ) and $\mathcal{M}$ a subspace of $M / R$. Then every weakly uniformly liftable mapping $f: \mathcal{M} \rightarrow M^{\prime} / R^{\prime}$ is continuous.

Proof Let $\phi: Z \rightarrow M^{\prime}$ be a weak uniform lifting of $f$. Note that $\mathcal{M} \subseteq \mathrm{st}_{M / R}(Z)$. By Propositions 2.2 and 2.4 the canonical bijection

$$
h: Z /\left(R \cap Z^{2}\right) \rightarrow \mathrm{st}_{M / R}(Z)
$$

is a homeomorphism. Since $\phi$ is a uniform lifting of $f \circ h: h^{-1}[\mathcal{M}] \rightarrow M^{\prime} / R^{\prime}$, by Proposition $4.1 f \circ h$ is continuous. Hence $f$ is also continuous. 
Lemma 4.3 Let $X$ be a dense subspace of a Hausdorff space $\tilde{X}, Y$ a subspace of a compact Hausdorff space $\tilde{Y}$, and $f: X \rightarrow Y$ a continuous mapping. Then there exists an extension $\tilde{f}: \tilde{X} \rightarrow \tilde{Y}$ of $f$ which is continuous at every point of $X$.

Proof (It is convenient to use nonstandard analysis at every step.) Let $F$ be the closure of $f$ in $\tilde{X} \times \tilde{Y}$. Since $X$ is dense in $\tilde{X}$ and $\tilde{Y}$ is compact, $\operatorname{dom} F=\tilde{X}$. Since $f$ is continuous, $F \cap(X \times \tilde{Y})=f$. Let $\tilde{f}: \tilde{X} \rightarrow \tilde{Y}$ be any mapping such that $\tilde{f} \subseteq F$; then $\tilde{f}$ is continuous at every point of $X$.

Lemma 4.4 Let $X$ be a nonempty closed subspace of metrizable space $\tilde{X}$. Then there exists a mapping $r: \tilde{X} \rightarrow X$ such that $r(x)=x$ for every $x \in X$ and which is continuous at every point of $X$.

Proof Let $d$ be any compatible metric on $\tilde{X}$. For every $y \in \tilde{X}$ choose $r(y) \in X$ so that $d(y, r(y)) \leq 2 d(y, X)$.

Lemma 4.5 Let $X$ be a Lindelöf subspace of a Tychonoff space $\tilde{X}$ and $Y$ a subspace of a compact Hausdorff space $\tilde{Y}$. Assume that at least one of the spaces $X$ and $Y$ is separable and metizable. Then for every continuous mapping $f: X \rightarrow Y$ there exists an extension $\tilde{f}: \tilde{X} \rightarrow \tilde{Y}$ which is continuous at every point of $X$.

Proof In both cases there exist continuous mappings $g: X \rightarrow Z$ and $h: Z \rightarrow Y$ such that $Z \subseteq[0,1]^{\mathbb{N}}$ and $h \circ g=f$. (Note that every separable metrizable space embeds into $[0,1]^{\mathbb{N}}$.) Since $X$ is Lindelöf, by [1, Corollary 16] for every $n \in \mathbb{N}$ there exists an extension $\tilde{g}_{n}: \tilde{X} \rightarrow \mathbb{R}$ of $g_{n}=\operatorname{pr}_{n} \circ g$ which is continuous at every point of $X$. We may assume that $\tilde{g}_{n}: \tilde{X} \rightarrow[0,1]$. Let $\tilde{g}: \tilde{X} \rightarrow[0,1]^{\mathbb{N}}$ be such that $\tilde{g}_{n}=\operatorname{pr}_{n} \circ \tilde{g}$ for every $n \in \mathbb{N}$; it is easily checked that $\tilde{g}$ is an extension of $g$ which is continuous at every point of $X$.

Let $K$ be the closure of $Z$ in $[0,1]^{\mathbb{N}}$. By Lemma 4.4 there exists a mapping $r$ : $[0,1]^{\mathbb{N}} \rightarrow K$ such that $r(t)=t$ for every $t \in K$ and which is continuous at every point of $K$. By Lemma 4.3 we obtain an extension $\tilde{h}: K \rightarrow \tilde{Y}$ of $h$ which is continuous at every point of $Z$. It is clear that $\tilde{f}=\tilde{h} \circ r \circ \tilde{g}$ is an extension of $f$ which is continuous at every point of $X$.

Proof of Theorem 1.8 Case (1): By Theorem 1.7 there exists a weak uniform lifting $\psi: H \rightarrow M^{\prime}$ of $f$ such that $H$ is a hyperfinite subset of $M$. By Proposition 2.3 there exists a *metric $d$ on $M$ with $R=d^{-1}\left(\mu_{\mathbb{R}}(0)\right)$. Since $H$ is hyperfinite, by transfer there 
exists an internal mapping $r: M \rightarrow H$ such that $d(x, r(x))=\min \{d(x, y): y \in H\}$ for every $x \in M$. It is clear that $\phi=\psi \circ r$ is a uniform lifting of $f$.

Cases (2)-(4): Since $\mathcal{M}$ is $\kappa$-separable (apply Lemma 3.1 in cases (2) and (3)), $f[\mathcal{M}]$ is also $\kappa$-separable. By Theorem 3.3 there exist $\Pi_{1}^{0}(\kappa)$ equivalence relations $\tilde{R}$ and $\tilde{R}^{\prime}$ on $M$ and $M^{\prime}$ respectively such that $\mathcal{M} \subseteq M / \tilde{R}, f[\mathcal{M}] \subseteq M^{\prime} / \tilde{R}^{\prime}$, and such that the spaces $M / \tilde{R}$ and $M^{\prime} / \tilde{R}^{\prime}$ are compact. In view of Proposition 4.1, it suffices to check that there exists an extension $\tilde{f}: M / \tilde{R} \rightarrow M^{\prime} / \tilde{R}^{\prime}$ of $f$ which is continuous at every $\mu \in \mathcal{M}$. In case (3) there is nothing to prove. In cases (2) and (4) we apply Lemma 4.5. In both cases $\mathcal{M}$ is Lindelöf (note that every separable metrizable space is Lindelöf). In case (2) $f[\mathcal{M}]$ is Lindelöf (as a continuous image of Lindelöf space) and $M^{\prime} / R^{\prime}$ is metrizable (by Propositions 2.3 and 2.4), therefore, $f[\mathcal{M}]$ is separable and metrizable (as a Lindelöf subspace of metrizable space).

Example 4.6 Let $X$ be a separable Tychonoff space which is not Lindelöf [4, Examples 3.8.13]. Assume also that $X$ is not almost compact (e.g., consider $X \times\{0,1\}$ instead of $X$ ). (A Tychonoff space $X$ is said to be almost compact if $|\beta X \backslash X| \leq 1$.) By [3, Theorem 1.3] there exist a Tychonoff space $Y$ containing $X$ as a subspace and a continuous function $f: X \rightarrow \mathbb{R}$ such that there is no extension $\tilde{f}: Y \rightarrow \mathbb{R}$ of $f$ which is continuous at every point of $X$. By [3, remark on p. 908] we may assume that $f: X \rightarrow[0,1]$. Let $K$ be the closure of $X$ in $\beta Y$; note that $K$ is separable and compact. Assume that our nonstandard universe $\left(V(\Xi), V\left(^{*} \Xi\right), *\right)$ is $\kappa$-saturated with $\kappa>2^{|\beta Y|}$ and also that $\beta Y \in V(\Xi)$. We claim that the following continuous mappings are not uniformly liftable (see Example 1.4):

$$
\begin{gathered}
g:\left\{\mu_{\beta Y}(x): x \in X\right\} \rightarrow\left\{\mu_{[0,1]}(r): r \in[0,1]\right\}, \quad \mu_{\beta Y}(x) \mapsto \mu_{[0,1]}(f(x)), \\
h:\left\{\mu_{\beta Y}(y): y \in K\right\} \rightarrow\left\{\mu_{K}(y): y \in K\right\}, \quad \mu_{\beta Y}(y) \mapsto \mu_{K}(y) .
\end{gathered}
$$

Assume that $g$ is uniformly liftable; using Proposition 4.1, we obtain that there exists an extension $\hat{f}: \beta Y \rightarrow[0,1]$ of $f$ which is continuous at every point of $X$; then $\tilde{f}=\hat{f} \mid Y$ is also continuous at every point of $X$, a contradiction.

If $h$ is uniformly liftable, then there exists a mapping $r: \beta Y \rightarrow K$ such that $r(y)=y$ for every $y \in K$ and which is continuous at every point of $K$. By Lemma 4.3 there exists an extension $f^{\prime}: K \rightarrow[0,1]$ of $f$ which is continuous at every point of $X$. Define $\tilde{f}: Y \rightarrow[0,1]$ by $\tilde{f}=\left(f^{\prime} \circ r\right) \mid Y$; it is clear that $\tilde{f}$ is an extension of $f$ which is continuous at every point of $X$, a contradiction. 


\section{References}

[1] A V Arhangel'skii, Relative topological properties and relative topological spaces, Topology Appl. 70 (1996) 87-99; doi:10.1016/0166-8641(95)00086-0.

[2] S Baratella, S-A Ng, Fixed points in the nonstandard hull of a Banach space, Nonlinear Analysis 34 (1998) 299-306; doi:10.1016/S0362-546X(98)00571-9.

[3] A Bella, IV Yaschenko, Lindelöf property and absolute embeddings, Proc. Amer. Math. Soc. 127 (1999) 907-913; doi:10.1090/S0002-9939-99-04568-2.

[4] R Engelking, General Topology, Heldermann Verlag, Berlin (1989).

[5] S Fajardo, HJ Keisler, Existence theorems in probability theory, Advances in Math. 120 (1996) 191-257; doi:10.1006/aima.1996.0037.

[6] S Fajardo, H J Keisler, Neometric spaces, Advances in Math. 118 (1996) 134-175; doi:10.1006/aima.1996.0020.

[7] E I Gordon, Nonstandard analysis and compact abelian groups, Sib. Math. J. 32 (1991) 197-209; doi:10.1007/BF00972766.

[8] E I Gordon, Nonstandard methods in commutative harmonic analysis, volume 164 of Translations of mathematical monographs, AMS (1997).

[9] C W Henson, The nonstandard hulls of a uniform space, Pacif. J. Math. 43 (1972) 115-137.

[10] C W Henson, Infinitesimals in functional analysis, from: "Nonstandard analysis and its applications", (N J Cutland, editor), London Mathematical Society Student Texts 10, Cambridge University Press (1988) 140-181.

[11] C W Henson, L C Moore, Nonstandard analysis and the theory of Banach spaces, from: "Nonstandard Analysis-Recent Developments", (A E Hurd, editor), Lecture Notes in Mathematics 983 (1983) 27-112; doi:10.1007/BFb0065334.

[12] W A J Luxemburg, A general theory of monads, from: "Applications of Model Theory to Algebra, Analysis and Probability", (W A J Luxemburg, editor), Holt, Rinehart and Winston (New York) (1969) 18-86.

[13] J Mlček, P Zlatoš, Some Ramsey-type theorems for countably determined sets, Arch. Math. Logic 41 (2002) 619-630; doi:10.1007/s001530100129.

[14] M Ziman, P Zlatoš, Hyperfinite dimensional representations of spaces and algebras of measures, Monatsh. Math. 148 (2006) 241-261; doi:10.1007/s00605-005-0375-3.

IICO-UASLP, Av. Karakorum 1470, Lomas 4ta Session, San Luis Potosí SLP, 78210 Mexico appyshchev@hotmail.com

Received: 23 September 2009 Revised: 12 December 2010 